\title{
The Modeling of Human-Structure Interaction
}

\author{
Huixuan Han and Ding Zhou
}

\begin{abstract}
The coupled model of human and structure system has been developed by considering the standing human body as an elastic non-uniform column with the distributed mass, damping and stiffness. The governing differential equations of the human-beam system and the human-plate system are, respectively, derived by using the Lagrange equation. A two-section elastic column is used to simulate the vibration of the human body. The dynamic characteristics of the human-structure interaction are analyzed by the use of the complex mode theory. The influence of the human standing on the beams or the plates on vibration characteristics of the coupled systems is investigated in detail. The numerical results are compared with the experimental ones existed in the literature, good agreement has been achieved. Therefore, the reasonability and correctness of the present model have been demonstrated.
\end{abstract}

Index Terms - Body model, dynamic characteristic, dynamic differential equations, human-structure coupled system.

\section{INTRODUCTION}

In recent years, an increasing number of problems related to human-induced vibrations of floors, footbridges, assembly structures and stairs are reported. There are several reasons for that, such as: (1) improved mechanical properties of structural materials leading to reduced structural mass, (2) increased length of structural spans leading to increased slenderness, and (3) aesthetic design requirements for eye-catching 'transparent' structural forms. The factors mentioned above usually lead to very lightweight structures with light damping. In such a case, the vibration excited by the crowd's activity should be considered because it results in the uncomfortable feeling of the presence crowd and/or results in the damage of the structure. Therefore, through the study on the human occupants-structure interaction, not only the safety problems of structure could be solved, but also the human's comfort problems under the structure vibration could be reduced.

The existing knowledge of human-structure interaction can be summarized as two key aspects: (1) how structural vibrations can influence forces induced by human occupants, and (2) how human occupants influence the dynamic properties of civil engineering structures. When the influence of human occupants on the dynamic properties of civil engineering structures is considered, occupants are often modeled just as additional mass to the structure (Fig. 1(a)). This model has been widely accepted for a long time.

Manuscript received December 6, 2016; revised May 2, 2017. The financial supports from the National Natural Science Foundation of China (11372127) and the Key Program of Natural Science Research of Jiangsu Provincial University, China (12KJA580002) are greatly acknowledged.

Huixuan Han and Ding Zhou are with the College of Civil Engineering, Nanjing Tech University, No. 30 Puzhou Road(S), China (e-mail: hankatherine1991@126.com, dingzhou57@yahoo.com).
Naturally, such a model leads to a frequency decrease. However, experimental study demonstrates that occupants also have the potential to increase existing natural frequencies and even create new vibration modes [1], [2]. Furthermore, it is widely acknowledged that human occupants increase damping of civil engineering structures [3], [4]. This just as additional mass of modeling humans cannot explain observed increases of natural frequencies, or the appearance of additional natural frequencies. Furthermore, it is difficult to use this model to explain the significant increase in damping observed in real life. This insufficiency has led to the proposal of a single degree-of-freedom (SDOF) occupant model (Fig. 1(b)).

Modeling a human body as a SDOF system on a structure is simple and popular and the parameters of the body models, such as mass, stiffness, damping ratio and natural frequency for individuals have been defined through shaking-table experiments and the best curve fitting between the measured apparent mass and SDOF or TDOF models [5]-[8].

It is gradually realized that modeling the human body as an isolated SDOF system or a crowd as an isolated distributed SDOF system and then placing the SDOF body system on a structure maybe is not the best approach for the study of human-structure interaction, see Fig. 2(b). Instead, a standing person can be modeled as an elastic non-uniform column with the distributed mass, damping and stiffness placed on the structure and only the fundamental vibration mode of the column is considered (Fig. 1(c)). This finding could lead to the new coupled model of human-structure interaction.

The present study uses the Lagrange equation to derive the governing differential equations of the human-beam system and the human-plate system, respectively. A two-section elastic column is taken to simulate the vibration of the human body. Then a multi-degree of freedom coupled system is developed. The dynamic characteristics of the human-structure interaction are analyzed by the use of the complex mode theory. The influence of the human standing on the beams or the plates on vibration characteristics of the coupled systems is investigated in detail.

\section{THE GOVERNING DIFFERENTIAL EQUATIONS OF THE HUMAN-BEAM SYSTEM}

Consider bodies of height of $\mathrm{H}$ on a non-uniform slender beam system as shown in Fig. 2 where the same physical parameters for all bodies are assumed for the simplicity in the analysis however without losing the generality. A lot of experimental investigations show that the fundamental frequency of the body plays a main role in the human-structure coupled vibration. Therefore, only the fundamental mode of the body vibration is considered in the present analysis. Here, the mechanical property of the body 
is described by an elastic column with variable mass $m(y)$, stiffness $k(y)$ and damping $c(y)$ distributions. The displacement $u_{H j}(y, t)$ of the $j$ th body on the beam can be expressed as:

$$
\begin{aligned}
& u_{H j}(y, t)=u_{s}\left(x_{j}, t\right)+u_{R j}(y, t) \\
= & u_{s}\left(x_{j}, t\right)+u_{H R j}(t) \phi_{H}(y)
\end{aligned}
$$

in which,

$$
u_{s}(x, t)=\sum_{n=1}^{\infty} \phi_{n}(x) q_{n}(t)
$$

$u_{s}\left(x, t\right.$ is the displacement of the beam. $\phi_{n}(x)$ is the nth modal function of the beam. $u_{R j}(y, t)$ is the relative displacement of the jth body to the beam. $\phi_{H}(y)$ is the fundamental modal function of the body.

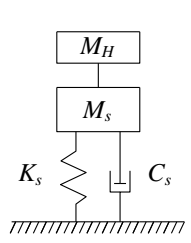

(a)

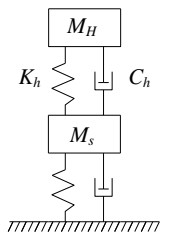

(b)

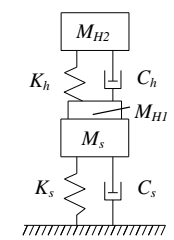

(c)
Fig. 1. Available models of human body on a SDOF platform.(a) Mass-only human-body model, (b) SDOF human-body model and (c) SDOF model with a non-vibrating mass.

The potential energy of the human-beam system is

$$
\begin{aligned}
U=U_{s} & +\sum_{j=1}^{J} U_{H j}=\sum_{n=1}^{\infty} q_{n}(t)^{2} \int_{0}^{L} \bar{k}(x)\left(\frac{d \phi_{n}(x)}{d x}\right)^{2} d x+ \\
& \frac{1}{2} \sum_{j=1}^{J} u_{H R j}{ }^{2} \int_{0}^{H} k_{j}(y)\left(\frac{d \phi_{H}(y)}{d y}\right)^{2} d y
\end{aligned}
$$

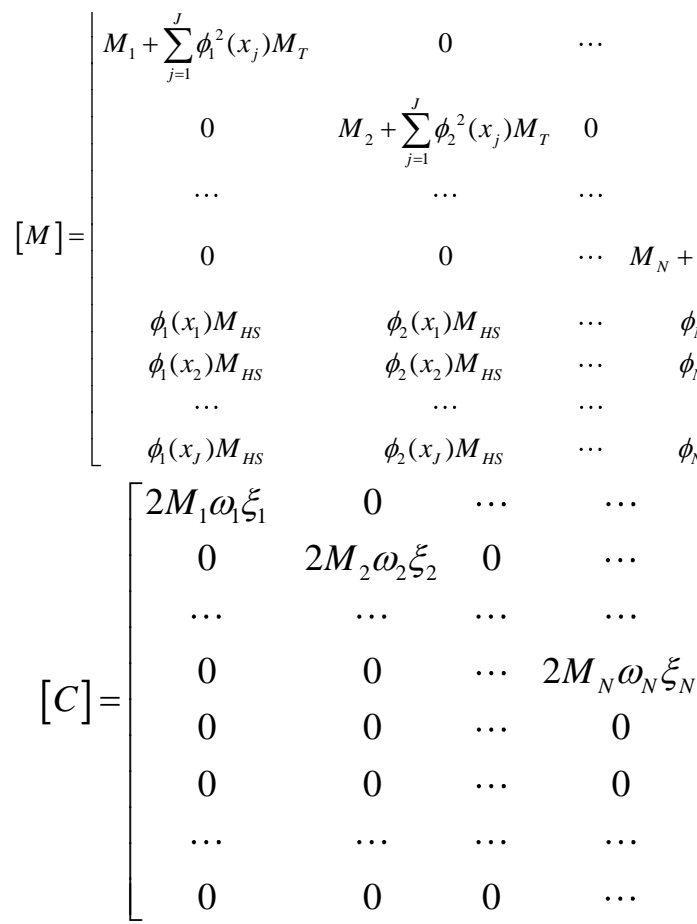

The kinetic energy of the coupled system is

$$
\begin{gathered}
T=T_{s}+\sum_{j=1}^{J} T_{H j}=\frac{1}{2} \sum_{n=1}^{\infty} \int_{0}^{L} \bar{m}(x)\left[\phi_{n}(x) \dot{q}_{n}(t)\right]^{2} d x+ \\
\frac{1}{2} \sum_{j=1}^{J} \int_{0}^{H} m(y)\left[\dot{u}_{s}\left(x_{j}, t\right)+\dot{u}_{H R j}(t) \phi_{H}(y)\right]^{2} d y
\end{gathered}
$$

The dissipated energy of the coupled system is

$$
\begin{gathered}
R=R_{s}+\sum_{j=1}^{J} R_{H j}=\frac{1}{2} \sum_{n=1}^{\infty} \dot{q}_{n}(t)(t)^{2} \int_{0}^{L} \bar{c}(x) \phi_{n}(x)^{2} d x+ \\
\frac{1}{2} \sum_{j=1}^{J} \dot{u}_{H R j}(t)^{2} \int_{0}^{H} c(y) \phi_{H}(y)^{2} d y
\end{gathered}
$$

where $\bar{k}(x), \bar{m}(x)$ and $\bar{c}(x)$ is variable mass, stiffness and damping distributions of the beam, respectively.

For the free vibration, the Lagrange equation is:

$$
\begin{gathered}
\frac{d}{d t}\left(\frac{\partial T}{\partial \dot{q}_{n}}\right)+\frac{\partial U}{\partial q_{n}}+\frac{\partial R}{\partial \dot{q}_{n}}=0 \quad(n=1,2, \ldots, N), \\
\frac{d}{d t}\left(\frac{\partial T}{\partial \dot{u}_{H R j}}\right)+\frac{\partial U}{\partial u_{H R j}}+\frac{\partial R}{\partial \dot{u}_{H R j}}=0 \quad(j=1,2, \ldots, J)
\end{gathered}
$$

Substituting (2a)-(2c) into (3a) and (3b) gives the governing differential equations in a matrix form as follows:

$$
[M]\{\ddot{X}\}+[C]\{\dot{X}\}+[K]\{X\}=0
$$

in which:

$$
\{X\}=\left[q_{1}, q_{2}, \cdots, q_{N}, u_{H R 1}, u_{H R 2}, \cdots u_{H R J}\right]^{T}
$$

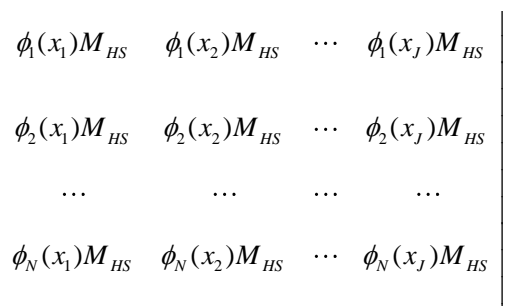
$\begin{array}{lllll}\phi_{N}\left(x_{1}\right) M_{H S} & M_{H} & 0 & \cdots & 0\end{array}$ $\phi_{N}\left(x_{2}\right) M_{H S}$

$\phi_{N}\left(x_{J}\right) M_{H S}$

$\begin{array}{llll}0 & M_{H} & \cdots & 0\end{array}$

$\begin{array}{cccc}\cdots & \cdots & \cdots & \cdots \\ 0 & 0 & \cdots & M_{H}\end{array}$

$$
\left.\begin{array}{cccc}
0 & 0 & \cdots & 0 \\
\ldots & 0 & \cdots & 0 \\
\ldots & \cdots & \cdots & \ldots \\
0 & 0 & \cdots & 0 \\
2 M_{H} \omega_{H} \xi_{H} & 0 & \cdots & 0 \\
0 & 2 M_{H} \omega_{H} \xi_{H} & \cdots & 0 \\
\ldots & \cdots & \cdots & \ldots \\
\ldots & \ldots & \cdots & 2 M_{H} \omega_{H} \xi_{H}
\end{array}\right]
$$




$$
[K]=\left[\begin{array}{cccccccc}
M_{1} \omega_{1}^{2} & 0 & \cdots & \cdots & 0 & 0 & \cdots & 0 \\
0 & M_{2} \omega_{2}^{2} & 0 & \cdots & \cdots & 0 & \cdots & 0 \\
\cdots & \cdots & \cdots & \cdots & \cdots & \cdots & \cdots & \cdots \\
0 & 0 & \cdots & M_{N} \omega_{N}^{2} & 0 & 0 & \cdots & 0 \\
0 & 0 & \cdots & 0 & M_{H} \omega_{H}^{2} & 0 & \cdots & 0 \\
0 & 0 & \cdots & 0 & 0 & M_{H} \omega_{H}^{2} & \cdots & 0 \\
\cdots & \cdots & \cdots & \cdots & \cdots & \cdots & \cdots & \cdots \\
0 & 0 & 0 & \cdots & \cdots & \cdots & \cdots & M_{H} \omega_{H}^{2}
\end{array}\right]
$$

where $M_{n}, \omega_{n}$ and $\xi_{n}(n=1,2, \ldots N)$ are the $i$ th modal mass, corresponding frequency and damping ratio of the bare beam, respectively. $\omega_{H}$ and $\xi_{H}$ are the individual body frequency and damping ratio, respectively.

$$
\begin{gathered}
M_{n}=\int_{0}^{L} \bar{m}(x) \phi_{n}(x)^{2} d x \quad(n=1,2, \ldots, N) \\
M_{T}=\int_{0}^{H} m(y) d y \\
M_{H S}=\int_{0}^{H} m(y) \phi_{H}(y) d y \\
M_{H}=\int_{0}^{L} m(y) \phi_{H}(y)^{2} d y
\end{gathered}
$$

where $M_{T}$ is the total mass of the body, $M_{H}$ is the modal mass of the body and $M_{H S}$ is the coupled mass of the body and structure. The coupled system leads to $N+J$ modes of vibration. Each mode is defined by its complex eigenvalue $\lambda_{r}(r=1,2, \ldots, N+J)$ and complex mode shape $\{\psi\}_{r}$.

The complex eigenvalue $\lambda_{r}$ defines the (damped) natural frequencies $f_{r}$ and the damping ratios $\zeta_{r}$ :

$$
f_{r}=\frac{1}{2 \pi}\left|\lambda_{r}\right|, \quad \zeta_{l}=\frac{-\operatorname{Re}\left(\lambda_{r}\right)}{\left|\lambda_{r}\right|} \quad(r=1,2, \ldots, N+J)
$$

When only one person stands on the beam and only the fundamental mode of the beam are considered, a simple physical model can be given in Fig. 3 where the mass $\left(M_{H S}-M_{H}\right)$ is attached to $M_{H}$ however makes the relative motion to $M_{s}$, i.e., the acceleration of ( $M_{H S}-M_{H}$ ) is equal to $\ddot{u}_{h}-\ddot{u}_{s}$. Comparing to Fig. 1(b), one can clearly see the difference between the present human-structure model and the conventional human-structure model.

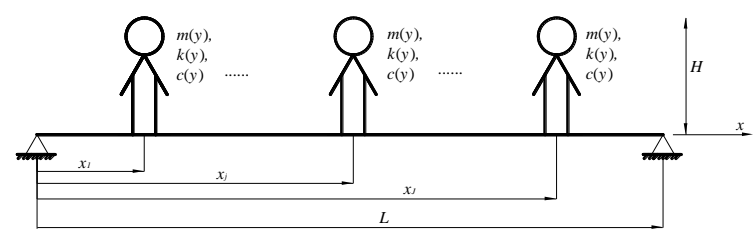

Fig. 2. The model of human-beam system.

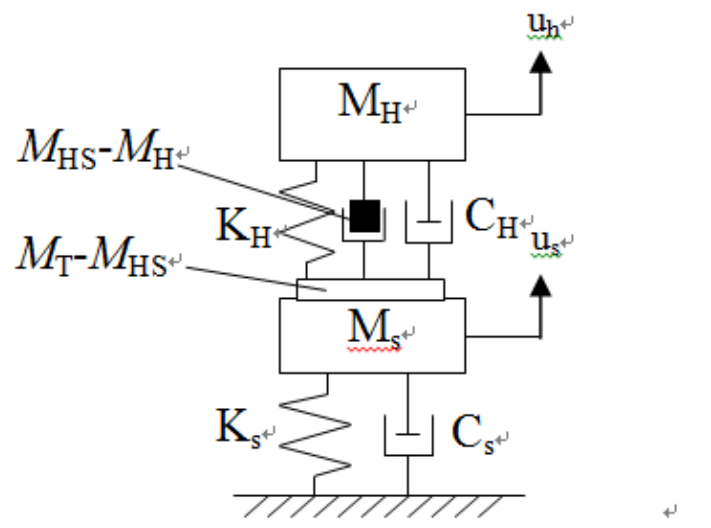

Fig. 3. The coupled physical model of single degree of freedom structure and single degree of freedom person

\section{THE GOVERNING DIFFERENTIAL EQUATIONS OF THE HUMAN-PLATE SYSTEM}

Consider bodies of height of $H$ on a uniform thin plate system as shown in Fig. 4. For simplicity and convenience in mathematical formulation, the following non-dimensional parameters are introduced:

$$
\alpha=2 x / a, \beta=2 y / b
$$

The mechanical property of the body can be described by an elastic column with the variable mass $m(y)$, stiffness $k(y)$ and damping $c(y)$ distributions as given in the last section. The displacement $w_{H j}(x, y, t)$ of the $j$ th body on the plate can be expressed as:

$$
w_{H j}(\alpha, \beta, t)=w\left(\alpha_{j}, \beta_{j}, t\right)+w_{R j}(z, t)
$$

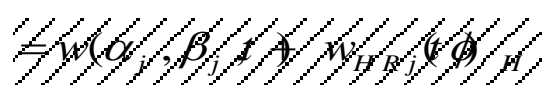

where $j$ means the $j$ th body on the beam and $J$ is the total number of body on the plate. $w(\alpha, \beta, t)$ is the plate displacement and can be represented as [9]:

$$
w(\alpha, \beta, t)=f_{w}^{1}(\alpha) f_{w}^{2}(\beta) \sum_{k=1}^{\infty} \sum_{l=1}^{\infty} P_{i}(\alpha) P_{j}(\beta) A_{k l}(t)
$$

where $P_{s}(\chi)(s=i, j ; \chi=\alpha, \beta)$ is the one-dimensional $s^{\text {th }}$ Chebyshev polynomial [9] which can be written in terms of cosine functions as follows: 


$$
P_{s}(\chi)=\cos [(s-1) \arccos (\chi)](s=1,2,3, \cdots)
$$

It should be noted that $f_{w}^{1}(\alpha)$ and $f_{w}^{2}(\beta)$ are the boundary characteristic functions corresponding to different boundary conditions, as given in Table I [9].

The potential energy of the human-plate system is

$$
\begin{aligned}
U=U_{s}+\sum_{j=1}^{J} U_{H j} & \\
=\frac{D}{2} \int_{-1}^{1} \int_{-1}^{1}\left[\left(\frac{4}{a^{2}} \frac{\partial^{2} w}{\partial \alpha^{2}}+\right.\right. & \left.\frac{4}{b^{2}} \frac{\partial^{2} w}{\partial \beta^{2}}\right)^{2}-\frac{32(1-\mu)}{a^{2} b^{2}}\left[\frac{\partial^{2} w}{\partial \alpha^{2}} \frac{\partial^{2} w}{\partial \beta^{2}}-\frac{\partial^{2} w}{\partial \alpha \partial \beta}\right] d \alpha d \beta \\
& +\frac{1}{2} \sum_{j=1}^{J} w_{H R j}(t)^{2} \int_{0}^{H} k_{j}(z)\left(\frac{d \phi_{H}(z)}{d z}\right)^{2} d z
\end{aligned}
$$

The kinetic energy of the coupled system is

$$
\begin{gathered}
T=T_{s}+\sum_{j=1}^{J} T_{H j}=\frac{a b \rho h}{8} \int_{-1}^{1} \int_{-1}^{1}\left(\frac{\partial w}{\partial t}\right)^{2} d \alpha d \beta+ \\
\frac{1}{2} \sum_{j=1}^{J} \int_{0}^{H} m(z)\left[\frac{d w\left(\alpha_{j}, \beta_{j}, t\right)}{d t}+\frac{d w_{H R j}(t)}{d t} \phi_{H}(z)\right]^{2} d z
\end{gathered}
$$

$$
R=R_{s}+\sum_{j=1}^{J} R_{H j}
$$

$=\frac{a b \rho h}{8} \int_{-1}^{1} \int_{-1}^{1} c(\alpha, \beta)\left(\frac{\partial w}{\partial t}\right)^{2} d \alpha d \beta+\frac{1}{2} \sum_{j=1}^{J} \dot{w}_{H \beta j}(t)^{2} \int_{0}^{H} c(z) \phi_{H}(z)^{2} d z$

For the free vibration, the Lagrange equation is:

$$
\begin{gathered}
\frac{d}{d t}\left(\frac{\partial T}{\partial \dot{A}_{k l}(t)}\right)+\frac{\partial U}{\partial A_{k l}(t)}+\frac{\partial R}{\partial \dot{A}_{k l}(t)}=Q_{n} \quad(k=1,2 \ldots K ; l=1,2 \ldots L) \\
\frac{d}{d t}\left(\frac{\partial T}{\partial \dot{u}_{H R j}}\right)+\frac{\partial U}{\partial u_{H R j}}+\frac{\partial R}{\partial \dot{u}_{H R j}}=Q_{j} \quad(j=1,2 \ldots J)
\end{gathered}
$$

Substituting (12a)-(12c) into (13a) and (13b) gives the governing differential equations in a matrix form as follows:

$$
[M]\{\ddot{X}\}+[C]\{\dot{X}\}+[K]\{X\}=0
$$

in which:

$$
\{X\}=\left[A_{11}, A_{12}, \cdots, A_{k l}, u_{H R 1}, u_{H R 2}, \cdots, u_{H R J}\right]^{T}
$$

The dissipated energy of the coupled system is

$$
[C]=\left[\begin{array}{cccccccc}
2 M_{1,1} \omega_{1,1} \xi_{1,1} & 0 & \cdots & \cdots & 0 & 0 & \cdots & 0 \\
0 & 2 M_{1,2} \omega_{1,2} \xi_{1,2} & 0 & \cdots & \cdots & 0 & \cdots & 0 \\
\cdots & \cdots & \ldots & \cdots & \cdots & \cdots & \cdots & \cdots \\
0 & 0 & \cdots & 2 M_{K, L} \omega_{K, L} \xi_{K, L} & 0 & 0 & \cdots & 0 \\
0 & 0 & \cdots & 0 & 2 M_{H} \omega_{H} \xi_{H} & 0 & \cdots & 0 \\
0 & 0 & \cdots & 0 & 0 & 2 M_{H} \omega_{H} \xi_{H} & \cdots & 0 \\
\cdots & \cdots & \cdots & \cdots & \cdots & \cdots & \cdots & \cdots \\
0 & 0 & 0 & \cdots & \cdots & \cdots & \cdots & 2 M_{H} \omega_{H} \xi_{H}
\end{array}\right]
$$

$$
[K]=\left[\begin{array}{cccccccc}
M_{1,1} \omega_{1,1}^{2} & 0 & \cdots & \cdots & 0 & 0 & \cdots & 0 \\
0 & M_{1,2} \omega_{1,2}^{2} & 0 & \cdots & \cdots & 0 & \cdots & 0 \\
\cdots & \cdots & \cdots & \cdots & \cdots & \cdots & \cdots & \cdots \\
0 & 0 & \cdots & M_{K, L} \omega_{K, L}^{2} & 0 & 0 & \cdots & 0 \\
0 & 0 & \cdots & 0 & M_{H} \omega_{H}^{2} & 0 & \cdots & 0 \\
0 & 0 & \cdots & 0 & 0 & M_{H} \omega_{H}^{2} & \cdots & 0 \\
\cdots & \cdots & \cdots & \cdots & \cdots & \cdots & \cdots & \cdots \\
0 & 0 & 0 & \cdots & \cdots & \cdots & \cdots & M_{H} \omega_{H}^{2}
\end{array}\right]
$$

in which,

$$
\begin{gathered}
M_{T}=\int_{0}^{H} m(z) d z \\
M_{H S}=\int_{0}^{H} m(z) \phi_{H}(z) d z \\
M_{H}=\int_{0}^{H} m(z) \phi_{H}^{2}(z) d z \\
M_{k, l}=\frac{a b \rho h}{4} \int_{-1}^{1} \int_{-1}^{1} f_{w}^{1}(\alpha)\left[f_{w}^{2}(\beta) P_{k}(\alpha) P_{l}(\alpha)\right]^{2} d \alpha d \beta \\
(k=1,2, \cdots K ; l=1,2 \cdots L)
\end{gathered}
$$

$$
\begin{array}{r}
\phi_{k, l}\left(\alpha_{j}, \beta_{j}\right)=f_{w}^{1}\left(\alpha_{j}\right) f_{w}^{2}\left(\beta_{j}\right) P_{k}\left(\alpha_{j}\right) P_{l}\left(\beta_{j}\right) \\
(k=1,2, \cdots K ; l=1,2 \cdots L ; j=1,2, \cdots J)
\end{array}
$$

The coupled system leads to $(K \times L+J)$ modes of vibration. Each mode is defined by its complex eigenvalue $\lambda_{r}(r=1$, $2, \ldots K \times L+J)$ and the corresponding complex mode shape $\{\psi\}_{r}$.

The complex eigenvalue $\lambda_{r}$ define the (damped) natural frequencies $f_{r}$ and the damping $\operatorname{ratios} \zeta_{r}$ : 


$$
f_{r}=\frac{1}{2 \pi}\left|\lambda_{r}\right|, \zeta_{l}=\frac{-\operatorname{Re}\left(\lambda_{r}\right)}{\left|\lambda_{r}\right|} \quad(r=1,2, \ldots, K \times L+J)
$$

TABLE I: BOUNDARY CHARACTERISTIC FUNCTION COMPONENTS FOR DIFFERENT BOUNDARY CONDITIONS

\begin{tabular}{lll}
\hline \hline Boundary conditions & $f_{w}^{1}(\alpha)$ & $f_{w}^{2}(\beta)$ \\
\hline F-F & 1 & 1 \\
F-S & $1-\alpha$ & $1-\beta$ \\
S-F & $1+\alpha$ & $1+\beta$ \\
S-S & $1-\alpha^{2}$ & $1-\beta^{2}$ \\
F-C & $(1-\alpha)^{2}$ & $(1-\beta)^{2}$ \\
C-F & $(1+\alpha)^{2}$ & $(1+\beta)^{2}$ \\
S-C & $(1+\alpha)(1-\alpha)^{2}$ & $(1+\beta)(1-\beta)^{2}$ \\
C-S & $(1-\alpha)(1+\alpha)^{2}$ & $(1-\beta)(1+\beta)^{2}$ \\
C-C & $(1-\alpha)^{2}(1+\alpha)^{2}$ & $(1-\beta)^{2}(1+\beta)^{2}$ \\
\hline \hline
\end{tabular}

Note: F: free edge, S:simply-supported edge, C: clamped edge.

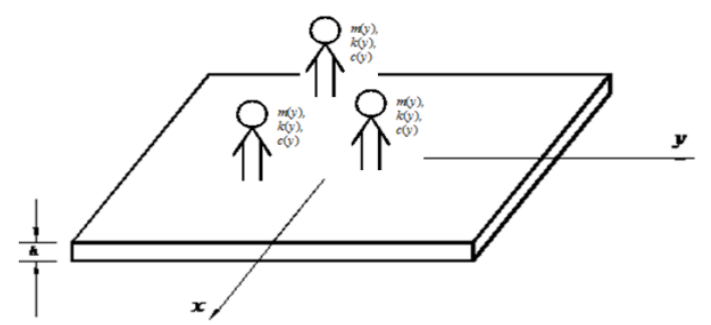

Fig. 4. The model of human-plate coupled system.

\section{MODAL PARAMETERS OF BODY}

According to the knowledge of biomechanics, the body can be simply modelled as a bar with two segments [10] as given in Fig. 5. The mass and longitudinal stiffness per unit length are the constants along each segment and the equation of axial motion is

$$
m_{i} \frac{\partial^{2} u_{i}}{\partial t^{2}}-k_{i} \frac{\partial^{2} u_{i}}{\partial x_{i}^{2}}=0, \quad(i=1,2)
$$

where $m_{i}$ and $k_{i}(i=1,2)$ are the mass and longitudinal stiffness densities of each segment. The solution of the above equation has the following form:

$$
u_{i}(x, t)=A \sin \left(\omega t+\varphi_{i}\right) \sigma_{i}(x), \quad i=1,2
$$

where $\omega$ is the radian frequency, $\varphi_{i}$ is the phase angle, $\sigma_{i}(x)$ is the mode shape function and $A$ is the magnitude of vibration. Using the end conditions of the body and the consistent conditions at the connection of two parts, the modal shape functions of the body are

$$
\begin{array}{ll}
\sigma_{1}\left(x_{1}\right)=D \sin b_{1} x_{1}, & 0 \leq x_{1} \leq L_{1}, \\
\sigma_{2}\left(x_{2}\right)=D\left[\sin b_{1} L_{1} \cos b_{2} x_{2}+\sqrt{\frac{m_{1} k_{1}}{m_{2} k_{2}}} \cos b_{1} L_{1} \sin b_{2} x_{2}\right], & 0 \leq x_{2} \leq L_{2}
\end{array}
$$

where $b_{1}=\omega \sqrt{k_{1} / m_{1}}, \quad b_{2}=\omega \sqrt{k_{2} / m_{2}}, D$ is the constant and determined by $|\sigma(x)|_{\max }=1$.
The frequency equation is

$$
\tan b_{1} L_{1} \tan b_{2} L_{2}=\sqrt{\frac{m_{1} k_{1}}{m_{2} k_{2}}}
$$

where $b_{2} L_{2}=b_{1} L_{2} \sqrt{k_{1} m_{2} /\left(k_{2} m_{1}\right)}$.

It is well known that to exactly give the mass and stiffness distributions is unpractical because the complicity of body. In the present study, four cases of mass and stiffness distributions of the body are studied and $L_{2}=L_{1}$ is taken. The corresponding modal parameters are given in Table II.

TABLE II: THE MODAL PARAMETERS OF THE BODY MODELS

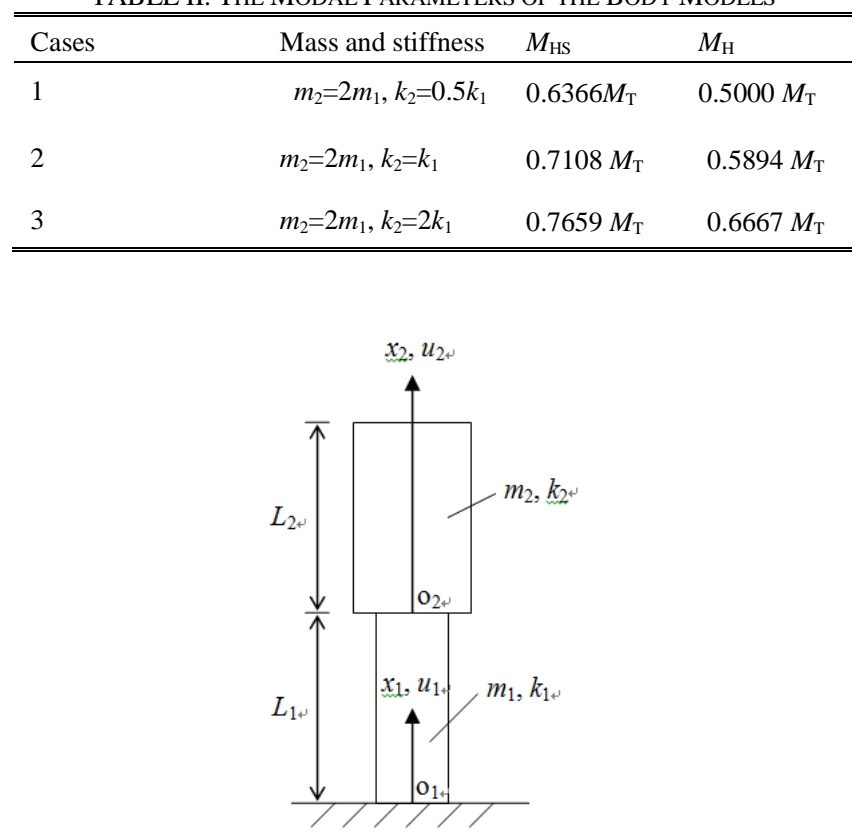

Fig. 5 . The model of body vibration in vertical direction.

\section{AN EXAMPLE}

To illustrate the use of the present model, an examples of human-beam interaction are analyzed. The example is a simply-supported beam of length $11.0 \mathrm{~m}$, width $1.25 \mathrm{~m}$ and thickness $0.35 \mathrm{~m}$ [11], [12]. The material properties of the beam are mass density, $\rho=2400 \mathrm{~kg} / \mathrm{m}^{3}$ and Young's modulus, $E=30 \times 10^{9} \mathrm{~N} / \mathrm{m}^{2}$. We only consider the first three vibration modes of the bare beam in the analysis. The natural frequency of the bare beam is $f_{1}=4.5 \mathrm{~Hz}$, $f_{2}=18 \mathrm{~Hz}$. The damping ratios of the bare beam are $\xi_{1}=0.3 \%, \quad \xi_{2}=0.075 \%$. The effective body mass contributing to vibration is considered to be the Case 3 in Table II with the fundamental natural frequency of $5.5 \mathrm{~Hz}$ [13], [14], the body mass of $70 \mathrm{~kg}$ and the body damping ratio of $\xi_{H}=0.42$. Figure 6 gives the damped natural frequencies and the damping ratios of the human-beam system with respect to the position, $x_{1}$ of single human at the beam in the interval $[0,11]$. Moreover, Fig. 7 gives the damped natural frequencies and the damping ratios of the 
human-beam system with respect to the human number $J$ in the interval $(1,23)$ where the human standing on the beam begins from the mid-span of the beam with a $0.5 \mathrm{~m}$ distance each other. In this case, the max number of human on the beam is 23. It is shown that the frequency of the first mode generally decreases with the increase of the human number and the frequency of the second mode and the third mode increases with the increase of human number. The damping ratio of the first mode to third mode increases with the increase of human number.

The numerical results are compared with the experimental ones given in the literature [12], [13], as presented in Table 3. It is shown that a good agreement has been achieved. Therefore, the reasonability and correctness of the present model have been demonstrated.
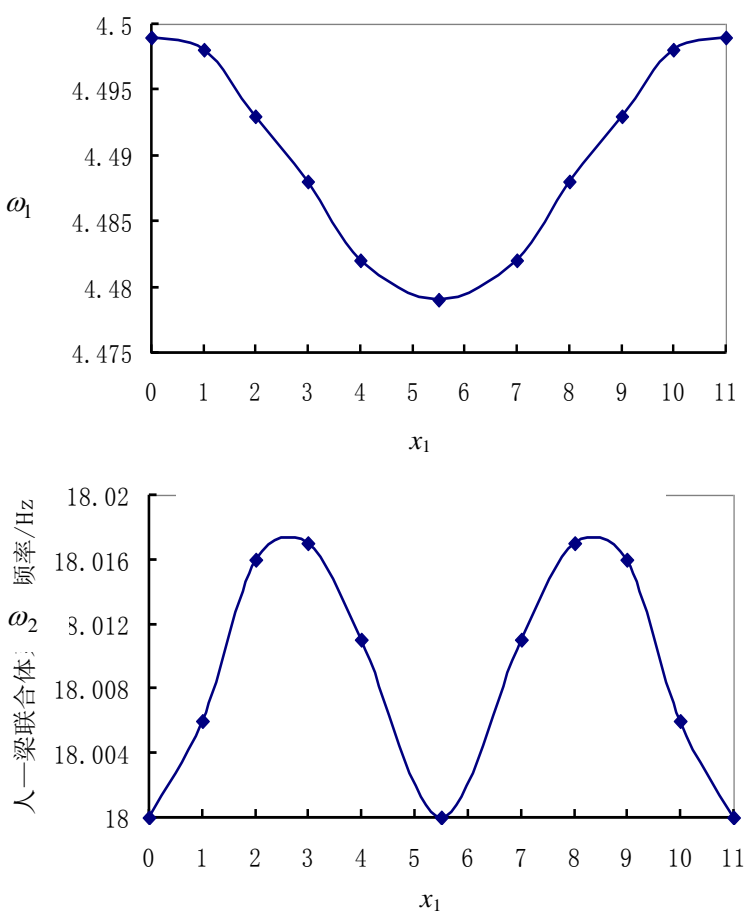

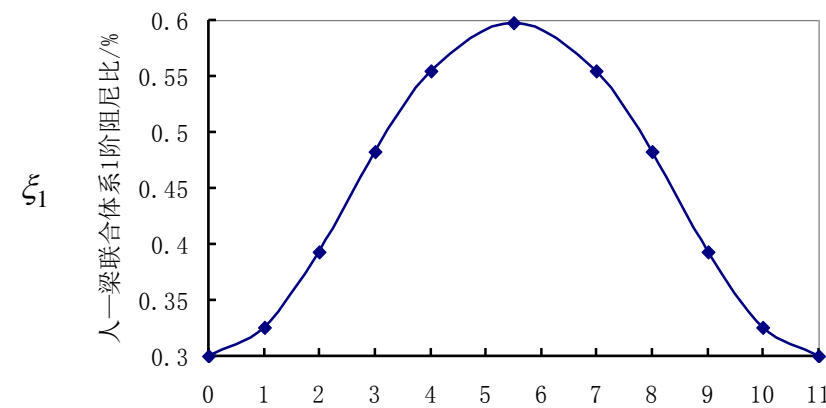

(a) The first mode

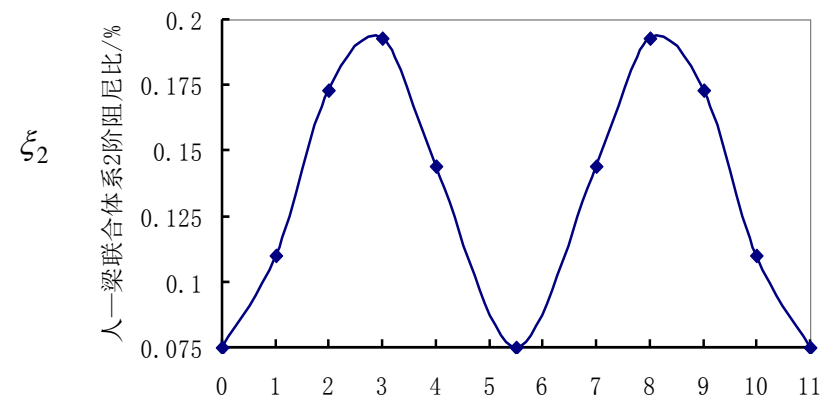

(b) The second mode

Fig. 6. The position effect of single human on the dynamic characteristics of human-beam system.

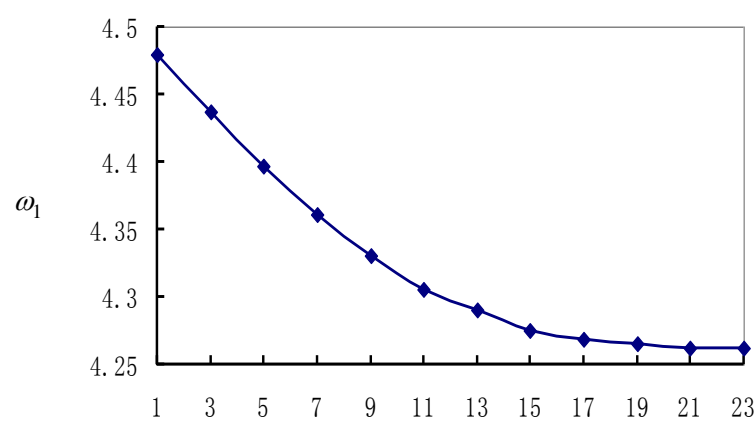

$J$

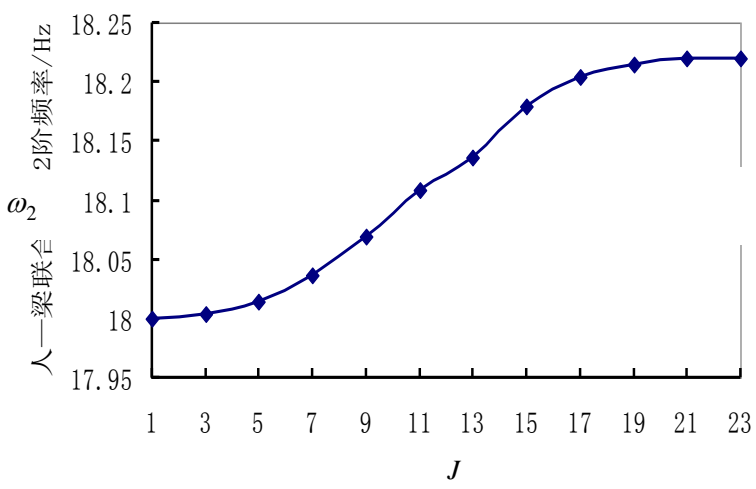

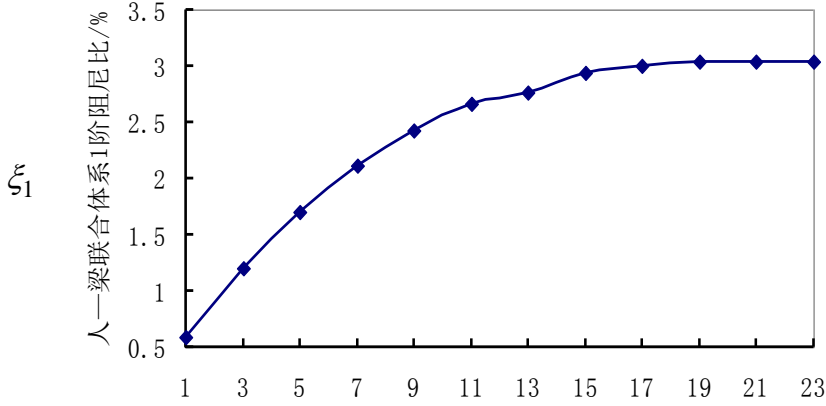

(a) The first mode

(a) The first mode

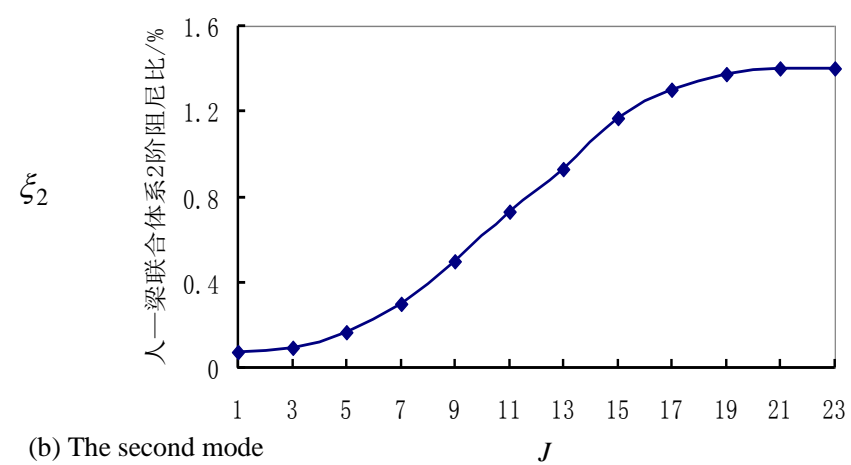

Fig. 7. The effect of human number on the dynamic characteristics of human-beam system. 
TABLE III: COMPARISONS OF THE EXPERIMENTAL RESULTS AND THE PRESENT NUMERICAL RESULTS

\begin{tabular}{lllll}
\hline \hline \multirow{2}{*}{$\begin{array}{l}\text { Human } \\
\text { number }\end{array}$} & \multicolumn{2}{l}{ The first frequency $/ \mathrm{Hz}$} & \multicolumn{2}{c}{ The first damping ratio } \\
1 & Present & Experiment & Present & Experiment \\
5 & $4.479 \mathrm{~Hz}$ & $\begin{array}{l}\text { decrease } \\
\text { trend }\end{array}$ & $0.60 \%$ & $0.6 \%$ \\
\hline \hline
\end{tabular}

\section{CONCLUSIONS}

This paper derived the governing differential equations of the human-beam system and the human-plate system by using the Lagrange equation, respectively. A two-section elastic column is used to simulate the vibration of the human body. The influence of the human standing on the beams or the plates on vibration characteristics of the coupled systems is investigated in detail. The main conclusions are summarised as follows:

1) A new human-structure model is developed, which is more reasonable than the conventional one. In the new model, the human body is considered as an elastic column on the structure. The difference between the present model and the conventional model can be clearly seen by comparing Fig. 3 and Fig. 2(b) where the term of the relative inertia force, $\left(M_{H S}-M_{H}\right)\left(\ddot{u}_{h}-\ddot{u}_{s}\right)$, is not existed.

2) The numerical results are compared with the experimental ones given in the literature, good agreement has been achieved. Therefore, the reasonability and correctness of the present model have been demonstrated.

3) In the analysis, the frequency of the first mode of human-beam system generally decreases with the increase of the human number. However, the frequency of the second mode and the third mode increases with the increase of the human number. The damping ratios of the first three modes increase with the increase of the human number. Therefore, the effect of human body on structural vibration characteristics is different for the different modes.

\section{REFERENCES}

[1] T. Ji, "Understanding the interactions between people and structures," The Structural Engineers, vol. 81, pp. 12-13, July 2003.

[2] J. H. Rainer and G. Pernica, "Damping of a floor sample," The Second Specialty Conference on Dynamic Response of Structures: Experimentation, Observation, Prediction and Control, pp. 859-873, Atlanta USA, 1981.

[3] A. Polensek, "Damping capacity of nailed wood-joist floors," wood science, vol. 8, pp. 140-151, March 1975.

[4] J. H. Rainer and G. Pernica, "Damping of a floor sample," in Proc. the Second Specialty Conference on Dynamic Response of Structures:
Experimentation, Observation, Prediction and Control, pp. 859-873, Atlanta USA, 1981.

[5] B. R. Ellis and T. Ji, "Human-structure interaction in vertical vibrations," in Proc. ICE: Structure and Buildings Conf., 1997, pp. 1-9.

[6] Y. Matsumoto and M. J. Griffin, "Dynamic response of the standing human body exposed to vertical vibration: influence of posture and vibration magnitude," Journal of Sound and Vibration, vol. 212, pp. 85-107, April 1998.

[7] M. J. Griffin, Handbook of Human Vibration, Academic Press, 1990.

[8] T. E. Fairley and M. J. Griffin, "The apparent mass of the seated human body: vertical vibration," Journal of Biomechanics, vol. 22, pp. 81-84, February 1989.

[9] N. J. Mansfield and M. J. Griffin, "Non-linearity in apparent mass and transmissibility during exposure to whole-body vertical vibration," Journal of Biomechanics, vol. 33, pp. 933-941, August 2000.

[10] D. Zhou, Three-dimensional vibration analysis of structural elements using Chebyshev-Ritz method, Science Press, 2007.

[11] L. Wei and M. J. Griffin, "Mathematical models for the apparent mass of the seated human body exposed to vertical vibration," Journal of Sound and Vibration, vol. 212, pp. 855-874, May 1998.

[12] R. Sachse, "Modeling effects of human occupants on modal properties of slender structures," The Structural Engineer, vol. 80, pp 21-22, March 2002.

[13] T. Ji, "A continuous model for the vertical vibration of the human body in a standing position," United Kingdom Informal Group Meeting on Human Response to Vibration, pp. 18-29, UK, 1995.

[14] R. Sachse, "The influence of human occupants on the dynamic properties of slender structures," Ph.D. dissertation, Dept. Civ. Struct. Eng., The Univ. of Sheffield, UK, 2002.

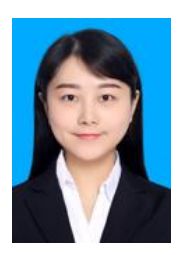

Huixuan Han was born in Changzhou, Jiangsu, China, and her birth date was February 24, 1991. She graduate with BE in 2013 in the College of Civil Engineering of Nanjing Tech University. She has been a Ph.D. candidate in the College of Civil Engineering of Nanjing Tech University since the year of 2013. In the present, her research area is mainly about human-structure interaction.

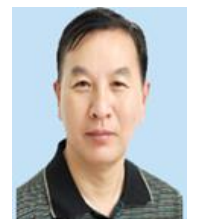

Ding Zhou, as her tutor, was born in Yancheng city of Jiangsu province in 1957 . He is working on static and dynamic analysis of composite structures, human-structure, soil-structure and fluid-structure coupling interaction as well as analysis of structure in temperature and fire fields. In addition to a committee of Journal of Applied Mathematical Modelling, Journal of ISRN Mechanical Engineering, Journal of Vibration Analysis, Measurement, and Control as well as Journal of Sound and Vibration, Ding Zhou has published more than 300 papers and other monographs, Application of Static Beam Function $\mathrm{n}$ Structural Vibration Analysis (China: Ding Zhou, 2013) and Three Dimensional Vibration Analysis of Structure Elements Based on Chebyshev-Litz Method (China: Ding Zhou, 2007) included. Ding Zhou, as a reviewer of 41 journals of SCI, also obtained the third prize of the National Defense Science and Technology $(1999,5)$ and the first prize of Jiangsu Province Science and Technology $(2014,5)$. On the other hand, he keeps cooperation with Department of Civil Engineering in University of Manchester, Department of Mechanical Engineering of Lake University, Canada and Howard University, USA. 